\title{
El Castillo de Capilla (Badajoz): un proyecto de puesta en valor (2012-2015)
}

\section{Diego Lucendo Díaz ${ }^{a}$, Tomás Torres González ${ }^{b}$, Luis Alejandro García García ${ }^{c}$ y Manuel Melero Serrano ${ }^{\mathrm{d}}$}

${ }^{a}$ Baraka Arqueologos S.L. C/ Granja de San Ildefonso 37, 4 J, 28051, Madrid, lucen2@hotmail.com, ${ }^{b}$ Baraka Arqueologos S.L C/Cervantes 39, 13247, San Carlos del Valle (Ciudad Real), tomastorresgonzalez@gmail.com, ${ }^{c}$ Baraka Arqueologos S.L Av.de Juan Pablo II 4, $2^{\circ}$ A, 45183, Las Ventas de Retamosa (Toledo), luisalejandrogg@hotmail.com, ${ }^{\mathrm{d} B a r a k a}$ Arqueologos S.L, Canal de Isabel II 9, $1^{\circ} \mathrm{C}, 28700$, San Sebastián de los Reyes (Madrid), mmeleroser@gmail.com.

\begin{abstract}
Resumen
El castillo de Capilla (Capilla, Badajoz) mostraba un grave estado de deterioro en sus fábricas a comienzos del siglo XXI, como consecuencia del abandono y la toma de medidas poco eficaces para su conservación. A partir de 2012, el Ayuntamiento de Capilla encargó el diseño un plan de actuaciones para frenar el deterioro del castillo y conseguir convertirlo en un activo para los recursos turísticos del municipio. El proyecto se centró en tres pilares (investigación, difusión y restauración) que han permitido devolver a la sociedad uno de los edificios más significativos de la historia medieval extremeña.
\end{abstract}

Palabras clave: castillo, fortificación, Edad Media, Almohade, excavación arqueológica.

\begin{abstract}
Capilla Castle (Capilla, Badajoz) showed a serious state of deterioration in its factories at the beginning of the 21st century as a result of neglect and ineffective measures taken for its conservation. As of 2012, the Capilla City Council commissioned the design of an action plan to halt the deterioration of the castle and manage to turn it into an asset for the municipality's tourist resources. The project focused on three pillars (research, dissemination and restoration) that have made it possible to return to society one of the most significant buildings in Extremadura's medieval history.
\end{abstract}

Keywords: castle, fortification, Middle Ages, Almohade, archaeological excavation. 


\section{Introducción}

El Castillo de Capilla se localiza en el cuadrante noroccidental del término municipal de Capilla (Badajoz), inmediatamente al oeste del actual núcleo urbano, en el extremo septentrional de la Sierra del Palenque. Se encuentra unos $2700 \mathrm{~m}$ al suroeste de la margen izquierda del río Zújar, y alrededor de $3900 \mathrm{~m}$ al oeste de su confluencia con el río Esteras. Ocupa la coronación de un promontorio rocoso conformado por afloramientos de esquistos silúricos fuertemente plegados y fracturados, que alcanza una altitud máxima de 565 m.s.n.m. Se sitúa, por tanto, alrededor de 85 $\mathrm{m}$ por encima de la cota del actual núcleo urbano de Capilla, y a algo más de $280 \mathrm{~m}$ de altura con respecto a la cota natural que presentaba el cauce del río Zújar con anterioridad a la construcción del embalse de La Serena. En la actualidad, la diferencia de cota entre el castillo de Capilla y la lámina de agua del embalse es de alrededor de $215 \mathrm{~m}$.

El cerro sobre cuya coronación asienta el castillo de Capilla presenta un perfil marcadamente cónico de laderas muy escarpadas, en especial por su flanco oriental, en el que se observan afloramientos rocosos con paredes verticales de hasta $40 \mathrm{~m}$ de altura. Una marcada depresión de algo más de $30 \mathrm{~m}$ de desnivel lo separa, al sur, del denominado Peñón del Pez, promontorio rocoso de $661 \mathrm{~m}$ de altitud sobre cuya ladera septentrional se conservan restos de obra fortificada de época protohistórica. Debido a su mayor altitud, el Peñón del Pez constituye, en términos de fortificación, un verdadero padrastro con respecto al castillo de Capilla, al que domina visualmente a una distancia mínima de $570 \mathrm{~m}$.

Debido a la combinación de todas estas circunstancias topográficas, el castillo de Capilla dispone de un amplio dominio visual del entorno, especialmente sobre la confluencia de los ríos Zújar y Esteras, que articulaban los pasos naturales de la zona tanto en dirección norte-sur como en dirección este-oeste. Sin embargo, la visibilidad del emplazamiento se encuentra sensiblemente mermada tanto hacia el sur, por donde resulta dominado por la Sierra del Palenque (cota 703 m.s.n.m.), como hacia el oeste, por donde se interpone la Sierra del Torozo (cota 846 m.s.n.m.).

La presencia humana en el término de Capilla se remonta hasta la prehistoria destacando la presencia de un conjunto de estelas de la Edad del Bronce, así como un importante conjunto de pintura esquemática distribuida por más de diez abrigos, descubiertos en parte por el Abate Breuil hace más de un siglo y cuya cronología podría abarcar desde el Neolítico hasta la primera Edad del Hierro (López, 2009).

En época prerromana, el territorio pertenecía a la Beturia Turdetana, una zona con grandes influencia del antiguo mundo tartésico pero también con fuertes influencias celtas (Berrocal-Rangel, 1995). A escasos $600 \mathrm{~m}$ al oeste del castillo, en el extremo norte de la sierra, se encuentra un gran asentamiento del Hierro II que perdura hasta época republicana, que actualmente recibe el nombre del Peñón del Pez. Tras la romanización, Capilla volverá a convertirse en el punto central de la comarca sucediendo al asentamiento en altura del Peñón del Pez, la población de Mirobriga Turdulorum, ubicada junto al río Zújar. Esta población a la que Plinio el Viejo la cita como oppida non ignobilia, confiriéndole una notable importancia político-administrativa, quedó reforzada con el título de Municipium Flavium, posiblemente en época de Vespasiano (Pastor y Pachón, 1992).

Ya en época islámica, en la zona de Capilla se asientan poblaciones beréberes, quienes llamarán a este territorio Fahs al-Ballut (Llano de las Bellotas) — que se corresponde con el valle de Los Pedroches (Córdoba) — y en el Monte de los bereberes Baranis, actuales sierras de Almadén y de Las Cabras. Se establece así una Cora con centro administrativo en Gafiq (Belalcázar, Córdoba). Capilla se constituyó en época califal en un nudo de comunicación en el que confluyeron las rutas que, procedentes de Córdoba y Sevilla, se dirigían hacia el norte (López, 2009). Aún así, el origen de la fortaleza de Capilla, nos es aún desconocido. Para algunos autores es durante el periodo de dominio almorávide cuando se construye el primitivo castillo (Ruibal, 1987). Según esta hipótesis, con la llegada de los almorávides y ante el aumento de las necesidades defensivas en la zona, la población islámica — hasta entonces situada más cerca del ríofue trasladada al emplazamiento actual del castillo, en lo alto de la Sierra del Palenque. Por otro lado, hasta el momento los restos documentales y arqueológicos no permiten corroborar esta hipótesis aunque el hallazgo de algún fragmento cerámico de época Omeya en las excavaciones arqueológicas podría indicar un origen anterior a este periodo.

Se ha identificado a Capilla como la Kabbal islámica (Gibello, 2007), una población perteneciente a la Kura de Fahs al Ballut citada por Al-Idrisi en la ruta de Córdoba a Miknasa. La primera cita documental cristiana conocida sobre Capilla data de 1182: el 30 de septiembre de ese año, Alfonso VIII firma en Alarcos un diploma que concede a la Orden de 
Calatrava el derecho al cobro de impuestos por el paso de los ganados que circulaban desde Toledo a Córdoba pasando por Capilla y Gahet (Belalcázar) (Gonzalez, 1960). A comienzos del siglo XIII Capilla tomaría gran relevancia como punto de defensa frente a la frontera cristiana acogiendo a gran parte de la población del entorno en su ladera. En 1226 Capilla pertenecía al reino de Baeza cuyo rey Al-Bayassi, con el fin de no enemistarse con el rey de Castilla Fernando III, llega a un acuerdo de paz a cambio de ceder las fortalezas de Burgalimar, Salvatierra y Capilla.

Sin embargo, cuando las tropas castellanas llegaron a la fortaleza de Capilla para tomar posesión de ella, sus habitantes se negaron a entregarla, lo que dio suceso a un largo asedio: “El rey a Castilla, (...), salió de Toledo con un pequeño ejército alrededor de la fiesta de Pentencostés en la era de 1264 [año 1226], y en principio con unos pocos puso sitio al noble castillo, fortísimo y populoso, de Capilla. Afirmado el asedio (...) con máquinas admirables” (Charlo, 1999, p. $80)$.

De la notable importancia estratégica del castillo de Capilla nos da cumplida cuenta la perseverancia de Fernando III en el asedio y la insistencia de su madre, doña Berenguela, en que no lo abandonase, pese a que la muerte del rey de Baeza proporcionaba a las tropas castellanas una inmejorable oportunidad para asaltar Córdoba. No obstante, el largo asedio y la ausencia de ayuda hace que los defensores de Capilla lleguen a un pacto con el rey de modo que "si el rey hispalense, que entonces estaba en Córdoba, quisiera prestarles ayuda dentro de ocho días de manera tal que obligaran a nuestro rey a retirarse del asedio, (...); de otra manera, entregarían a nuestro rey el castillo salvas las personas y los bienes muebles que pudieran llevarse" (Charlo, 1999). Pasado el plazo y tras la ausencia de ayuda, el rey castellano permitió a los habitantes de Capilla exiliarse a la vecina población de Gahet (Belalcazar), miestras que reparaba las defensas del castillo de Capilla y lo avituallaba con hombre y armamento para su correcta defensa.

La gestión del territorio, como pasó en gran parte de la meseta sur, fue cedida a las Ordenes Militares, de este modo el 9 de septiembre de 1236, Fernando III concedió a la Orden del Temple el castillo, el señorío y la villa de Capilla con todo su término, al que se sumó en diciembre de ese mismo año el de la vecina fortaleza de Almorchón. Las posesiones templarias con centro administrativo en Capilla se convirtieron en una extensa encomienda militar de gran importancia económica, principalmente ganadera. Del periodo templario apenas si tenemos documentación. En 1310, reducida la presencia militar al reino de Granada, la importancia militar de Capilla sería mínima y en el castillo residían únicamente el comendador, de nombre Juan Miguel con un sirviente. (Martínez Díez, 2001).

El siglo XIV será un periodo en el que la propiedad cambiará de manos en multitud de ocasiones, entre 1309 y 1320 estará bajo la Orden de Alcántara, volviendo a la Corona que lo cederá en 1344 al Concejo de aunque en 1346 también lo cederá el rey a Alfonso Fernández Coronel (Lopez, 2009), durante unos años existirán litigios hasta la muerte de este último en 1352 y la vuelta a la Corona. Nuevamente en 1370 cambiará de manos y pasará al Justicia Mayor del Rey Juan Nuñez de Villazán quién lo venderá en 1382 a Diego López de Estuñiga, Duque de Béjar, quienes mantendrán la propiedad hasta la unión de este ducado con el de Osuna en 1777. Posteriormente el edificio iría entrando en un abandono que no se revertirá hasta finales del siglo XX, momentos en los que empiezan las primeras intervenciones de restauración y conservación.

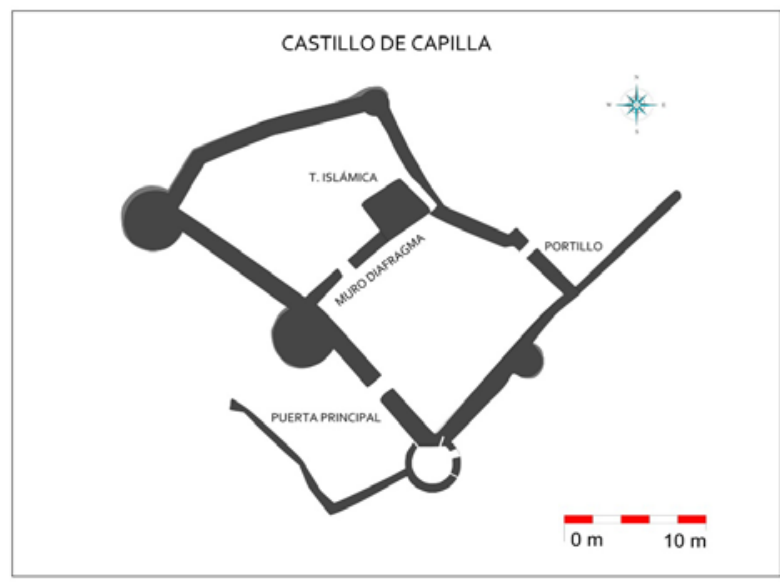

Fig. 1 Planta del castillo de Capilla 


\section{Intervenciones arqueológicas realizadas}

El castillo de Capilla conserva restos de obras significativas desde época islámica hasta el siglo XIX, correspondiéndose la imagen del edificio actual, casi en su totalidad, al periodo medieval y moderno cristiano. Su superficie construida no supera los $900 \mathrm{~m}^{2}$, quedando actualmente visibles los muros perimetrales, con restos de tres grandes torres circulares en su frente sur, una pequeña semicircular en el frente este y otra pequeña en la esquina noroeste. En su interior, se observa una división en dos espacios diáfanos mediante un gran muro que sirve de diafragma, quedando restos de diversas dependencias en cada espacio (Figs. 1 y 2).

Actualmente el castillo es propiedad municipal y en gran medida es el ayuntamiento el encargado de la gestión y conservación del edificio. En 2012 se encargó una estrategia global para la puesta en valor del edificio, que fuese más allá de las intervenciones puntuales y descoordinadas que se habían llevado hasta el momento. El objetivo de este plan era conseguir convertir al edificio en un recurso turístico y cultural del pueblo, sin dejar de lado la labor de investigación que llenara de contenido la visita turística y que evitara errores o daños irreversibles en la restauración del castillo.

Con estas premisas, se dividió el proyecto en tres ramas, que aunque se deberían ejecutar por profesionales diferentes, tendrían de estar en estrecho contacto a fin de crear sinergias que ayudasen a una correcta ejecución.

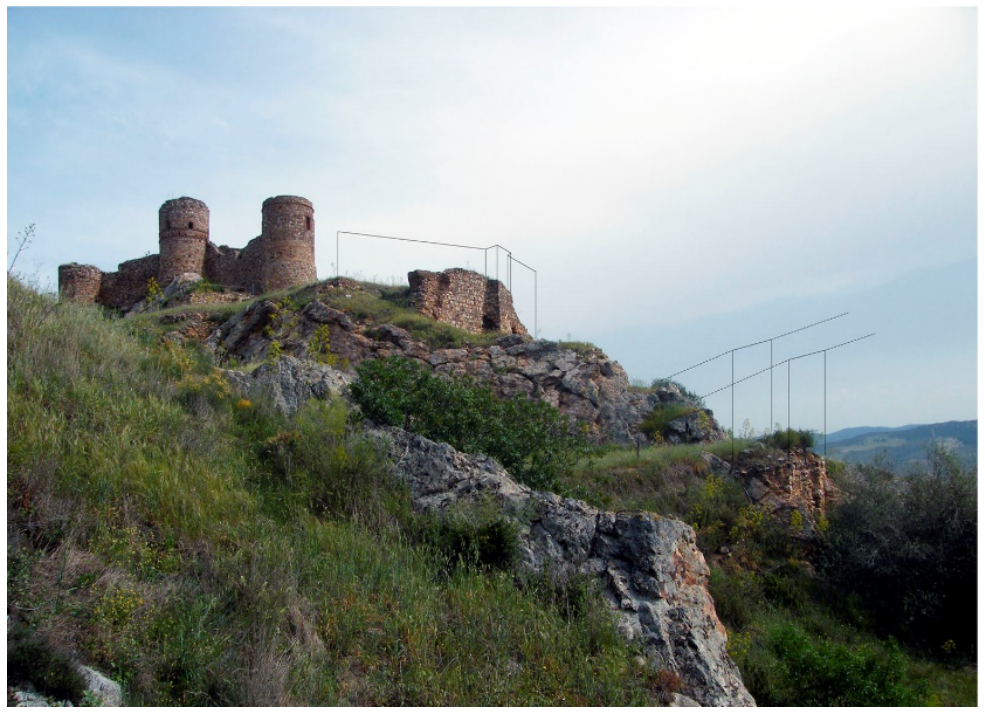

Fig. 2 Restos de los recintos amurallados que rodean al castillo

\subsection{Investigación}

El estudio en detalle de la historia del edificio y su entorno ha permitido conocerlo con más detalle. En primer lugar, se realizó una prospección superficial de todo el entorno del castillo. Las fuentes hablaban de un castillo fuerte y populoso, sin embargo, los estudios modernos habían reducido las dimensiones de la antigua Kabbal islámica al castillo y a los recintos amurallados que los rodeaban. Las prospecciones localizaron una importante puebla extramuros que tuvo su plenitud en época almohade, ubicada en la ladera oeste de la cresta que finaliza en el castillo. La prospección también aportó el hallazgo de nuevos tramos de las murallas que rodeaban la fortaleza, escondidas en las cárcavas del cerro y en puntos poco accesibles, estos pequeños tramos nos han ayudado a definir mejor el trazado de los recintos que rodeaban el castillo a la vez que han aportado datos constructivos de gran interés para su datación. De este modo, en uno de los tramos mejor conservados se observa en el acerado de tapial un despiece de sillería dibujado, similar a los documentados en una gran cantidad de fortalezas islámicas (Benquerencia, Reina) y fechado según los estudios de Rafael Azuar en el periodo Almohade.

Tras esta primera toma de contacto se procedió a una intervención arqueológica directa mediante el desarrollo de tres cursos de arqueología en verano (2012, 2013 y 2014). Los trabajos se dividieron en dos partes: 


\subsubsection{Interior del primer recinto del castillo}

Hasta la restauración del año 2015 era la única zona que poseía las condiciones necesarias para el trabajo debido a que deterioro del muro divisorio impedía transitar bajo él con seguridad. Este espacio con unos $310 \mathrm{~m}^{2}$ útiles y planta en forma de rectángulo irregular, se convierte en el primer cuerpo del castillo con las dos únicas entradas a este; la puerta principal al sur y un portillo en alto al norte. En su interior, las excavaciones arqueológicas sacaron a la luz parte de un patio enlosado de origen islámico que cubría un gran aljibe de dos naves del mismo periodo. Del periodo cristiano se documentaron los restos de una cuadra al este de la entrada y un gran espacio cuadrado en la esquina noroeste, así como pequeños espacios y reparaciones sobre el enlosado del patio que adscribimos al periodo bajomedieval.

Conjuntamente con la excavación de este primer recinto del castillo, se realizó una lectura de todos los paramentos del castillo, con el objetivo de comprender mejor el edificio y que esta información sirviese para las futuras intervenciones de restauración. Los resultados de este estudio han llegado a documentar al menos cinco periodos constructivos desde época islámica hasta el final de la Edad Moderna. Este estudio, muy útil en la restauración del castillo en 2015, posibilitó que no se eliminasen para siempre algunos elementos arquitectónicos que explican el edificio y su evolución. A grandes rasgos, ya que este no es el momento de realizar una descripción pormenorizada de la lectura de paramentos, las grandes fases constructivas se corresponden con:

- $\quad 1^{\mathrm{o}}$ Fase. Periodo islámico. En este periodo se construye la primera fortaleza y los recintos que la rodean, de los que se conservan varios restos de murallas construidos a base de zócalo de mampostería y alzado de tapial, al igual que alguna torre cuadrada de similar construcción. El castillo islámico presentaba una planta diferente al actual, más alargado en dirección noroeste-suroeste como constatan los restos conservados y posiblemente con una anchura similar. En el lateral suroeste se conserva un tramo de muralla de tapial de tierra sobre zócalo de mampostería cuarcítica que formó el límite suroeste de la antigua fortaleza. En el interior del edificio destacan su gran aljibe de dos naves y una pequeña torre, de la que se conserva su base, que integra un aljibe y que en su posición actual no tiene ninguna función. Los restos conservados nos muestran que la mayor parte de la obra islámica era en tapial, pudiendo datar en época almohade al menos el anillo de muralla más exterior a tenor de la decoración de sus tapiales con dibujos de despiece de sillería.

- $\quad 2^{\circ}$ Fase. Fernando III el Santo. La toma del Capilla tras catorce semanas de asedio causó grandes desperfectos, lo que conllevó una serie de reparaciones pues la crónica latina nos dice "El rey, reparadas las ruinas y roturas de los muros según el tiempo permitió y protegido el castillo con vituallas, armas, máquinas y hombres de guerra, volvió a Toledo" (Charlo, 1999). Las reparaciones documentadas en esta fase se corresponden con una serie de chapados en mampostería de tapiales islámicos ya muy deteriorados en el momento de la reparación, tanto en el muro suroeste del castillo como en diversos tramos de las murallas. Estos chapados debieron mostrar desde su inicio una notable debilidad y concuerdan con la imagen de una reparación apresurada.

- $\quad 3^{\circ}$ Fase. Siglo XIV. Es la tercera gran etapa constructiva del castillo, ya que al periodo templario únicamente se le puede adscribir la pequeña torre semicircular maciza que se encuentra en el muro suroeste. Durante el siglo XIV el edificio presenta una fuerte transformación con la construcción del frente actual del castillo, seguramente durante la tenencia del castillo por parte del Concejo de Toledo a mediados de siglo, aunque la gran diversidad de propietarios en este periodo no nos hacen descartar que la obra fuese ejecutada por varios propietarios. Constructivamente, el frente del castillo de Capilla con el uso de ladrillo en torres y puerta, al estilo mudéjar, recuerda a obras toledanas como la puerta del Vado, la torre de la Reina de Toledo o a la torre de la Vela del castillo de Maqueda (Fig. 3). Esta nueva reforma consideramos que pretendía ser general en todo el edificio, pero la obra debía realizarse sobre la antigua fortificación islámica sin que ésta perdiera su funcionalidad, realizando la sustitución de los distintos tramos de lienzos en diferentes fases consecutivas. Esto es, al menos lo que se deduce de la presencia de numerosas suturas constructivas verticales con cambio de aparejo en todo el tramo correspondiente a esta fase. La vuelta del castillo a la Corona y los diferentes cambios de propietarios hasta que finalmente llega a manos del Duque de Béjar en 1382, así como la ausencia de una necesidad defensiva clara, supone la paralización de esta fase constructiva. 
- $\quad 4^{\circ}$ Fase. Siglos XV-XIX. Las obras realizadas por la casa de Béjar son de peor calidad que las realizadas en el siglo XIV y de un carácter más puntual. En la fachada del edificio pertenece a esta fase la reconstrucción de los cuerpos superiores de la torre de la esquina sureste con una ejecución mucho menos cuidada que la que se puede observar en la torre central. Por otro lado, gran parte de los tramos noreste y noroeste del castillo pertenecen a esta fase del Duque de Béjar, que levantó estos lienzos por daños estructurales, como se aprecia en la esquina noreste, donde tras un derrumbe se reconstruye la misma sobre un arco de descarga de ladrillo para evitar cargar el peso sobre la obra antigua. A este periodo pertenecen gran parte de los edificios cuyos restos se observan en el interior del castillo. Esta fase es muy prolongada, por lo que se observan reformas posteriores, como recrecidos de algunos lienzos, especialmente el noreste, dejando fosilizados tramos de adarve y almenados.

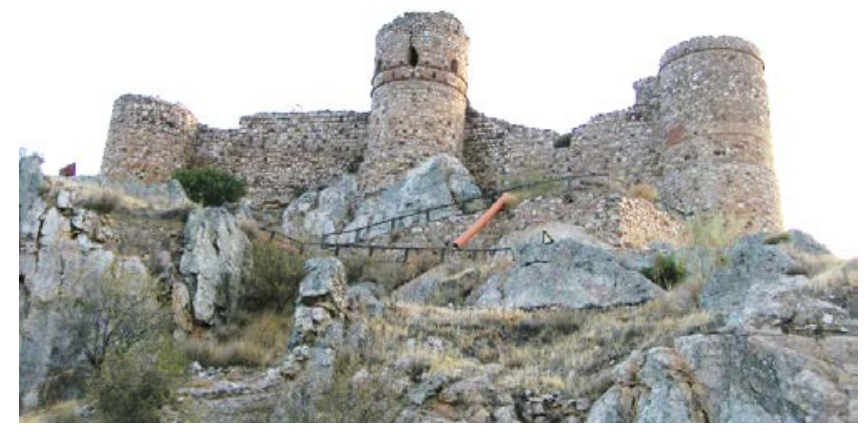

Fig. 3 El característico frente del castillo, se corresponde en gran medida, a obras del siglo XIV

\subsubsection{Puebla asociada al castillo}

Uno de los grandes hallazgos de este proyecto fue la localización de los restos de una puebla extramuros, con una superficie superior a las dos hectáreas. En estas campañas se han documentado, casi en su totalidad, dos viviendas pertenecientes al periodo almohade asociadas a una calle escalonada o rampa mulera. Estas viviendas de tamaño mediano, se dividen en tres o cuatro espacios en la planta inferior, donde se aprecian la presencia de pequeños patios y podrían tener una segunda planta o azotea a tenor de la presencia de una escalera de mampostería en una de las viviendas. Sus muros están construidos con mampostería de piedra cuarcítica trabada con barro y muy posiblemente la parte superior sería de tapial, aunque no lo hemos podido documentar, las techumbres de la mayor parte de los espacios eran de teja curva.

Todos los materiales arqueológicos documentados en el interior de las viviendas pertenecen al periodo islámico, especialmente al almohade. En el momento del asedio de Capilla en 1226, esta parte del poblado debió de sufrir sobremanera, como indican el gran número de puntas de flecha que se encuentran en sus rellenos, especialmente en los derrumbes de tejas. Tras la conquista de Capilla y la expulsión de sus habitantes, esta parte del poblado quedó abandonada quedando de ese modo fosilizado un poblado de época almohade de gran interés arqueológico.

Esta primera fase de investigación proporcionó una gran cantidad de información arqueológica muy valiosa, que corroboraban a las fuentes y empezaba a darle una dimensión más acorde del valor y potencial de este conjunto arqueológico. Pero para que este valor transcendiera de los investigadores a la sociedad era necesario dar el siguiente paso.

\subsection{Divulgación de los resultados}

La divulgación de los resultados de las campañas arqueológicas tenía un doble fin; devolver a la sociedad parte de los recursos invertidos en estos trabajos y conseguir concienciar a la población del municipio el gran recurso que supone para ellos el conjunto arqueológico de Capilla. Con este fin se llevaron a cabo diversas actividades divulgativas unidas a 
los cursos de arqueología, como exposiciones temporales (Fig. 4), visitas guiadas y una importante presencia en prensa y medios de comunicación. La gran acogida de todas las actividades realizadas impulsó al ayuntamiento a solicitar ayuda a la Junta de Extremadura para llevar a cabo una labor de consolidación y restauración de las partes más frágiles del castillo que serviría de impulso al resto de las actividades que se estaban realizando.

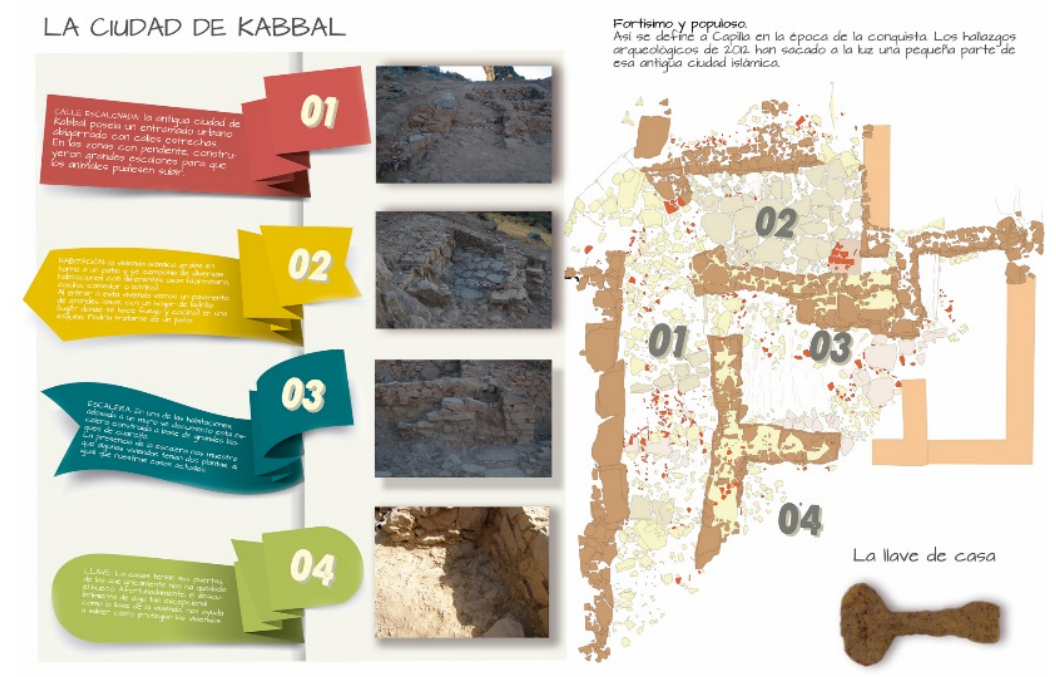

Fig. 4 Panel sobre la puebla almohade de una de las exposiciones

\section{Trabajos de restauración}

El proyecto de restauración, redactado por el arquitecto Alfonso Navarro Muñoz, se llevó a cabo en el año 2015. Esta intervención tuvo una importancia clave en el edificio debido a que su resultado final permitió que el castillo fuese visitable al público y que se pudiesen realizar intervenciones arqueológicas en todo el interior del mismo. Dentro del conjunto de restauraciones realizadas, destacaremos las dos intervenciones claves para su recuperación:

En primer lugar, se restauró el muro divisorio interior del castillo que posee el único acceso al recinto superior y a las torres del frente sureste (Fig. 5). El estado de este muro era muy precario, había perdido gran parte de su cara externa y la mitad del arco de la puerta, por lo que transitar por ella era muy peligroso. Su restauración posibilitó el acceso de personas al recinto superior con seguridad.

En segundo lugar, el acondicionamiento de los adarves del frente sureste con el acceso a dos de las torres, ha dado al interés turístico del castillo un gran empuje, pues desde las torres, aparte de elementos de interés arquitectónico, son miradores donde el visitante puede contemplar el impresionante paisaje de la cola del embalse de la Serena y las sierras que lo circundan.
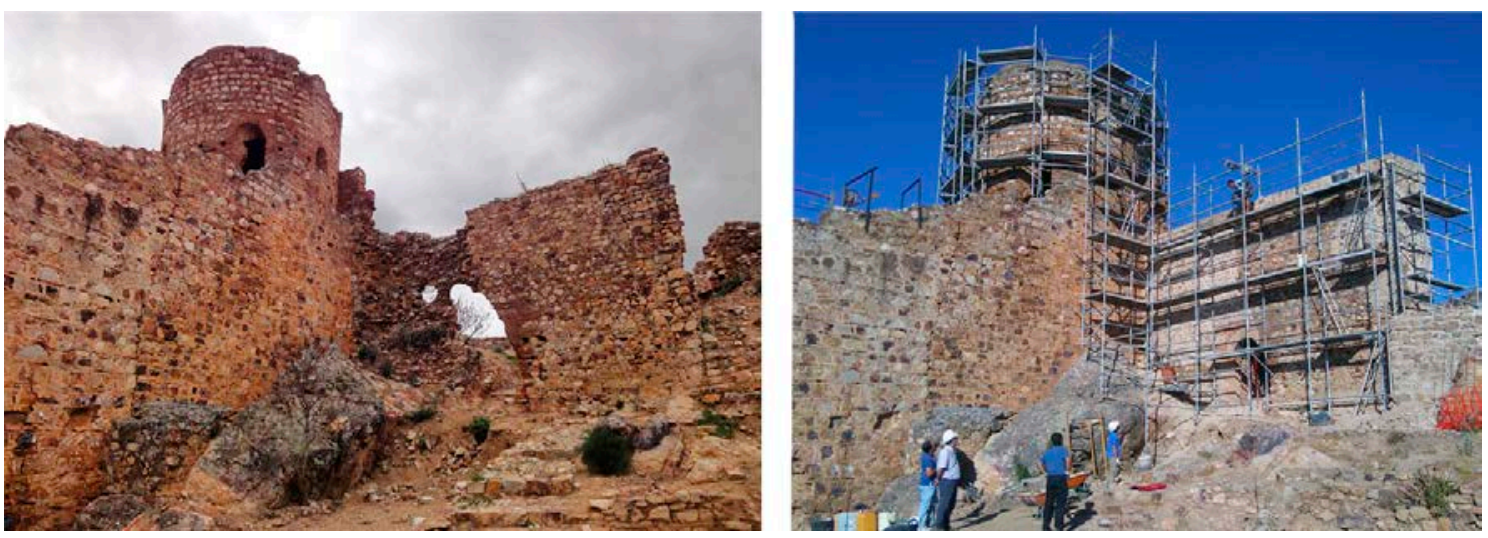

Fig. 5 Proceso de restauración y consolidación de las partes en riesgo de desaparición 


\section{Conclusiones}

El conjunto arqueológico del castillo de Capilla ya había sido objeto de intervenciones de restauración con anterioridad al año de 2012, sin embargo, la inmensa mayoría habían sido puntuales, aisladas y no respondían a un plan general de intervención.

A raíz del inicio de las campañas de intervención promovidas por el Ayuntamiento de Capilla a partir de 2012 y hasta la restauración del año 2015, se desarrolló una estrategia amplia, de forma coordinada con el consistorio, basada en la excavación de diversas zonas, interiores y exteriores del castillo, que permitieron documentar diversas estructuras como patio empedrado, cuadra o caballeriza y aljibe en el primer caso, y una pequeña superficie de la puebla en el segundo.

En el presente artículo, se ha pretendido adelantar alguno de los resultados obtenidos. La investigación arqueológica desarrollada hasta 2014 sentó las bases del estudio arqueológico realizado en el castillo y permitió plantear la campaña de restauración de 2015.

Los resultados de las distintas campañas arqueológicas irán viendo la luz de forma paulatina, así como el estudio de paramentos realizado, que ha permitido comprender la evolución constructiva del castillo a lo largo de sus sucesivas fases y remodelaciones.

Todos los trabajos que actualmente se realizan en el inmueble se deben a lo planteado en aquellos años. El fín último debe ser avanzar en el estudio y conservación de esta imponente fortaleza para que sirva de revulsivo al propio municipio y a la comarca. La difusión realizada hasta el momento, ha servido para que la población local sea más consciente de la enorme riqueza patrimonial que posee el municipio de Capilla.

\section{Referencias}

Berrocal Rangel, L. (1995). La Beturia. Definición y caracterización de un territorio prerromano. Cuadernos Emeritenses, 9, 151204.

Charlo Brea, L. (1999). Crónica Latina de los Reyes de Castilla. Biblioteca de Clásico Latinos Medievales. Barcelona: Editorial Akal.

Gibello Bravo, V. M. (2007). El poblamiento islámco en Extremadura: territorio, asentamientos e itinerarios. Mérida: Junta de Extremadura.

Gonzalez González, J. (1960). El reino de Castilla en la época de Alfonso VIII. Madrid: 3 vols.

Martínez Díez, G. (2001). Los Templarios en los Reinos de España. Barcelona: Planeta.

Pastor Muñoz, M., y Pachón Romero, J. A. (1992). Mirobriga. Excavaciones Arqueológicas en el “Cerro del Cabezo”, Capilla (Badajoz). Campañas de 1987-1988. Mérida: Consejería de Educación y Cultura.

Ruibal Rodríguez, A. (1987). Castillo de Capilla. Castillos de España 94, 19-30. Madrid: Asociación Española de Amigos de los Castillos. 